\title{
Tectonic plates in 3D mantle convection model with stress- history-dependent rheology
}

\section{CURRENT STATUS: UNDER REVIEW}

Earth, Planets and Space $\underline{\underline{0}}$ Springer

Takehiro Miyagoshi

Kaiyo Kenkyu Kaihatsu Kiko Yokohama Kenkyujo

Masanori Kameyama

IPU Kantaiheiyo Daigaku Tanki Daigakubu

kameyama@sci.ehime-u.ac.jpCorresponding Author

ORCiD: https://orcid.org/0000-0003-1739-2242

Masaki Ogawa

Tokyo Daigaku Daigakuin Sogo Bunka Kenkyuka Kyoyo Gakubu

\section{DOI:}

10.21203/rs.3.rs-18967/v1

\section{SUBJECT AREAS}

Astronomy

\section{KEYWORDS}

Mantle convection, 3D numerical model, Plate Tectonics, Stress-history-dependent rheology 


\section{Abstract}

Plate tectonics is a key feature of the dynamics of the Earth's mantle. By taking into account the stress-history-dependent rheology of mantle materials, we succeeded in realistically producing tectonic plates in our numerical model of mantle convection in a three-dimensional rectangular box. The calculated lithosphere is separated into several pieces (tectonic plates) that rigidly move. Deformation of the lithosphere caused by the relative motion of adjacent plates is concentrated in narrow bands (plate margins) where the viscosity is substantially reduced. The plate margins develop when the stress exceeds a threshold and the lithosphere is ruptured. Once formed, the plate margins persist, even after the stress is reduced below the threshold, allowing the plates to stably move over geologic time. The vertical component of vorticity takes a large value in the narrow plate margins. Secondary convection occurs beneath old tectonic plates as two-dimensional rolls with their axes aligned to the direction of plate motion. The surface heat flow decreases with increasing distance from divergent plate margins (ridges) in their vicinity in the way the cooling half-space model predicts, but it tends towards a constant value away from ridges as observed for the Earth because of the heat transport by the secondary convection.

\section{Full-text}

Due to technical limitations, full-text HTML conversion of this manuscript could not be completed. However, the manuscript can be downloaded and accessed as a PDF.

\section{Figures}

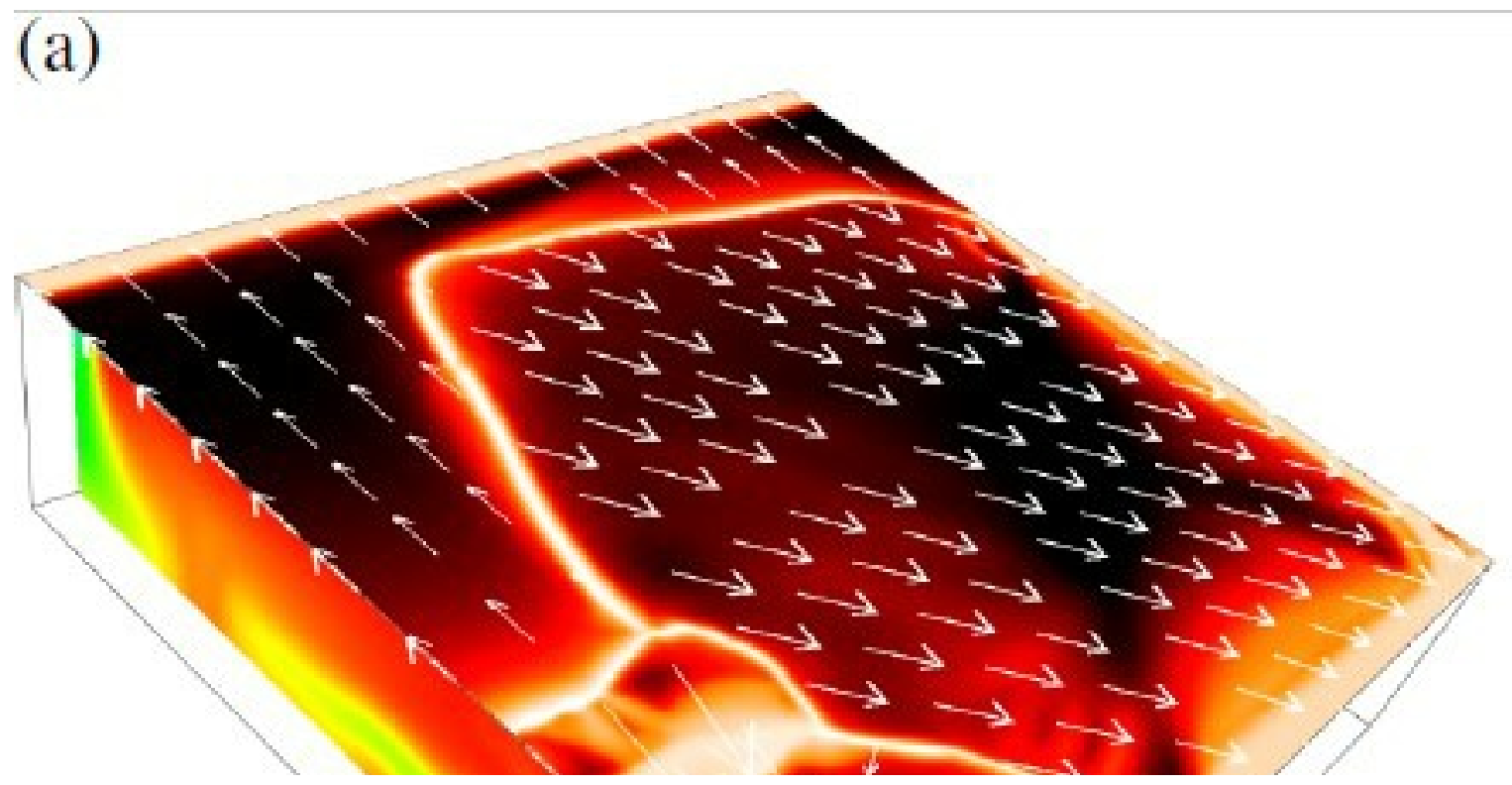




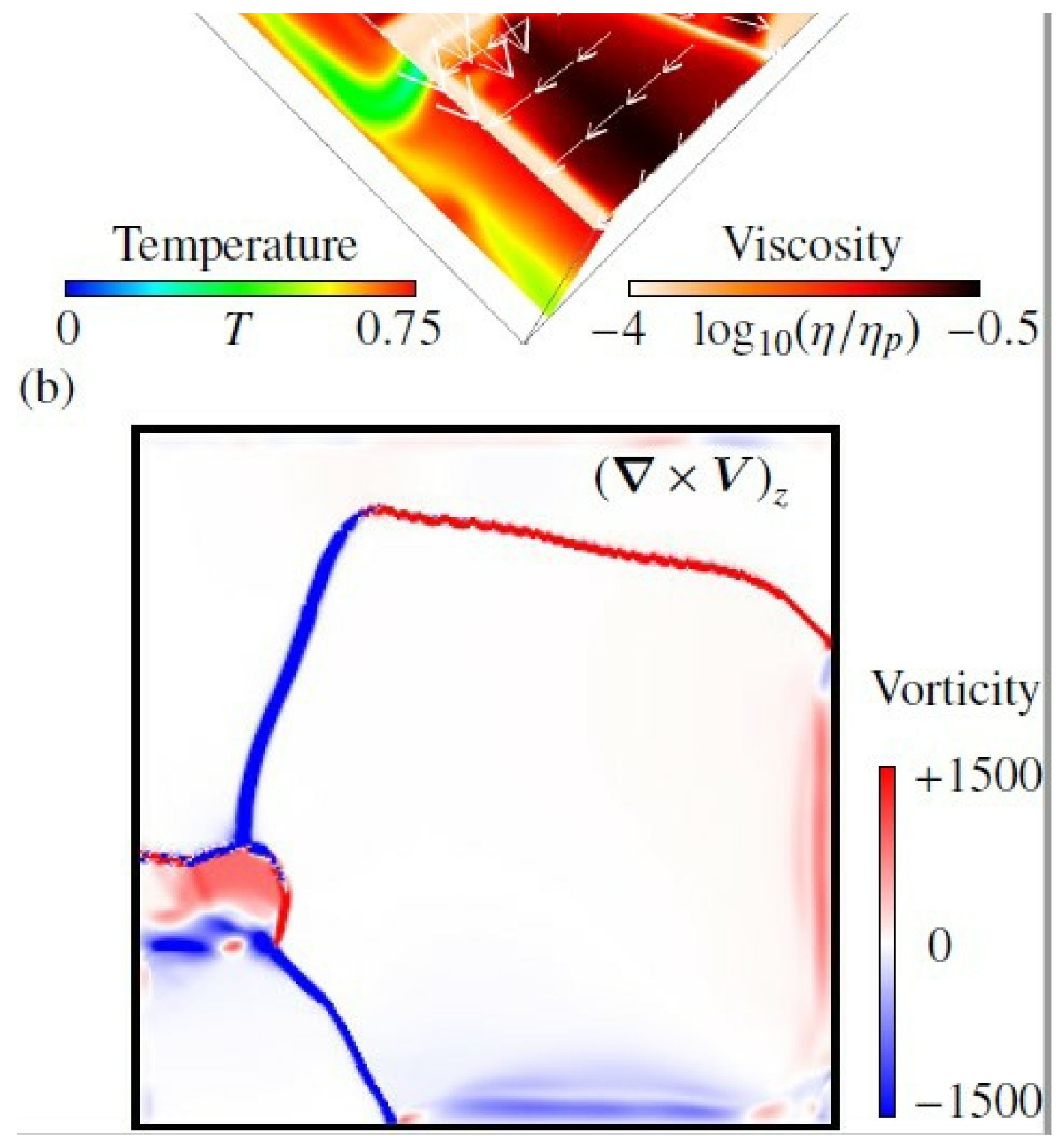

Figure 1

The overall three-dimensional structure of thermal convection obtained in our numerical model with stress-history-dependent rheology. (a) The distribution of temperature $\mathrm{T}$ in a vertical cross section and that of viscosity $\eta$ (colour) and velocity $\vee$ (arrows) on the surface.

The value of $\eta$ is normalised by $\eta p$, the viscosity of intact material at the surface. (b) The distribution of the vertical component of vorticity $(\nabla \times V) z$ on the surface.

(a)

(b) 


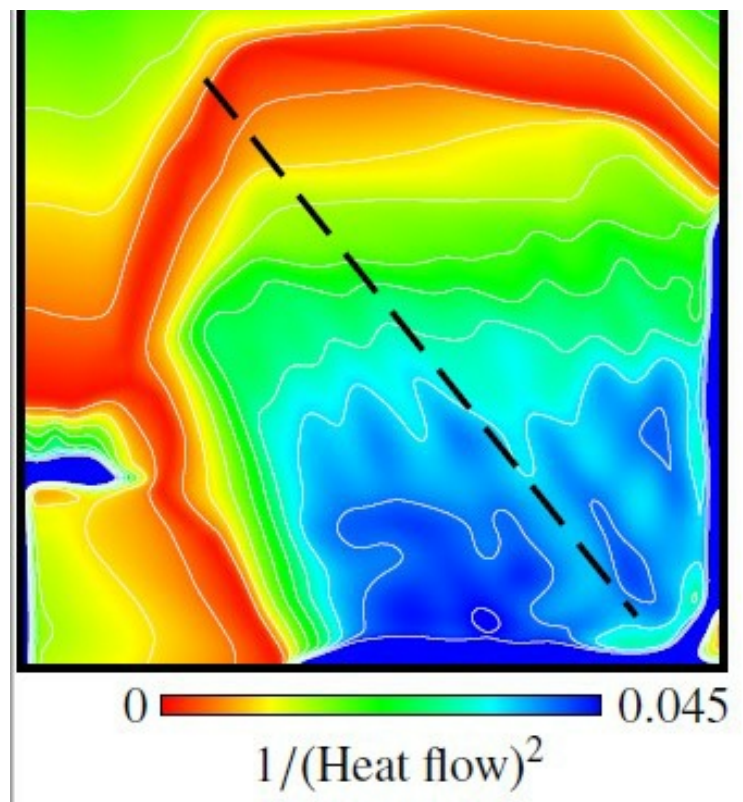

$1 /(\text { Heat flow })^{2}$

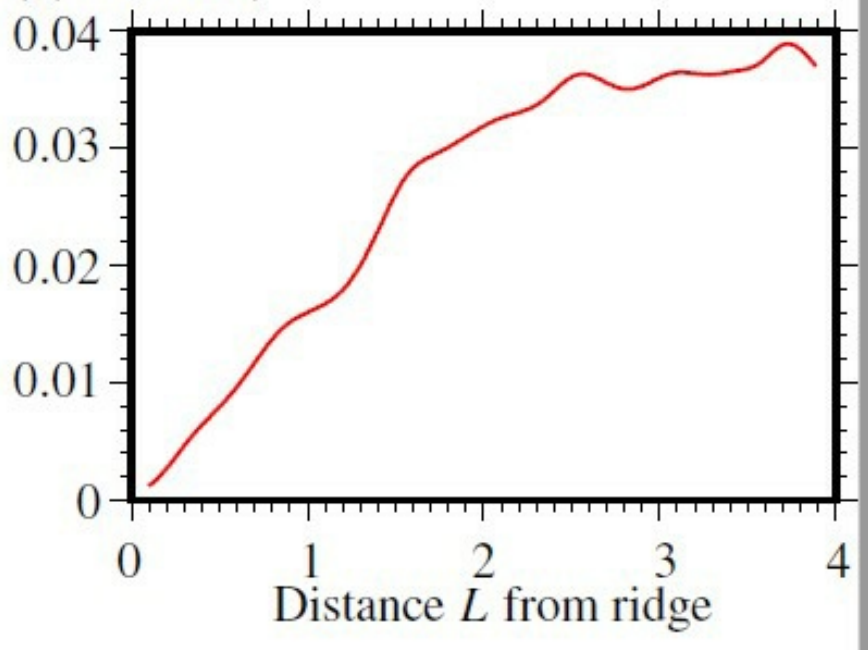

(c)

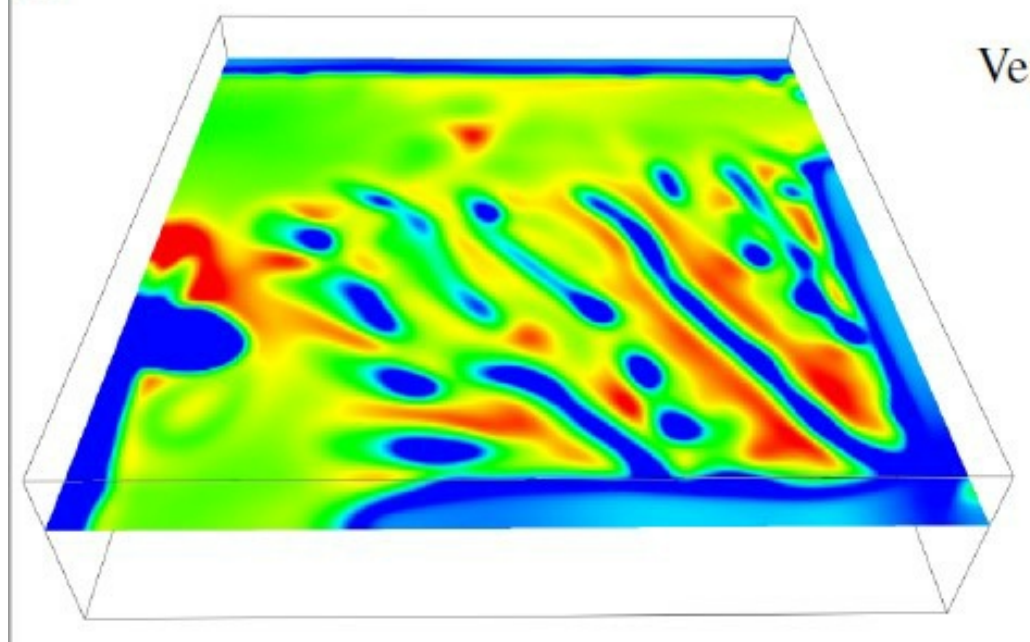

Vertical velocity

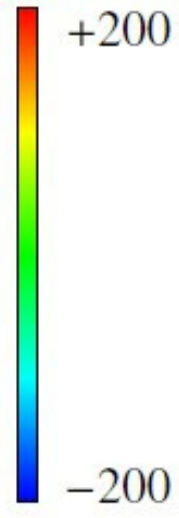

Figure 2

The lateral variations in the surface heat flow and convecting flow structure. (a) The distribution of the inverse square of the surface heat flow shown in both colour and white contour lines. (b) The plot of the inverse square of heat flow at the surface as a function of the distance $L$ from the divergent plate boundary (ridge) along a horizontal track indicated by the dashed line in (a). (c) The distribution of vertical component of velocity Vz on the horizontal plane at the height of $z=0.672$. Figure 3 . The time evolution of the surface plate motions. Snapshots of the distributions of viscosity $\eta$ (colour) and velocity $\vee$ (arrows) in a horizontal plane along the surface at the elapsed times indicated in the figure. The values of $\eta$ are normalised by $\eta p$, the viscosity of intact material at the surface. 


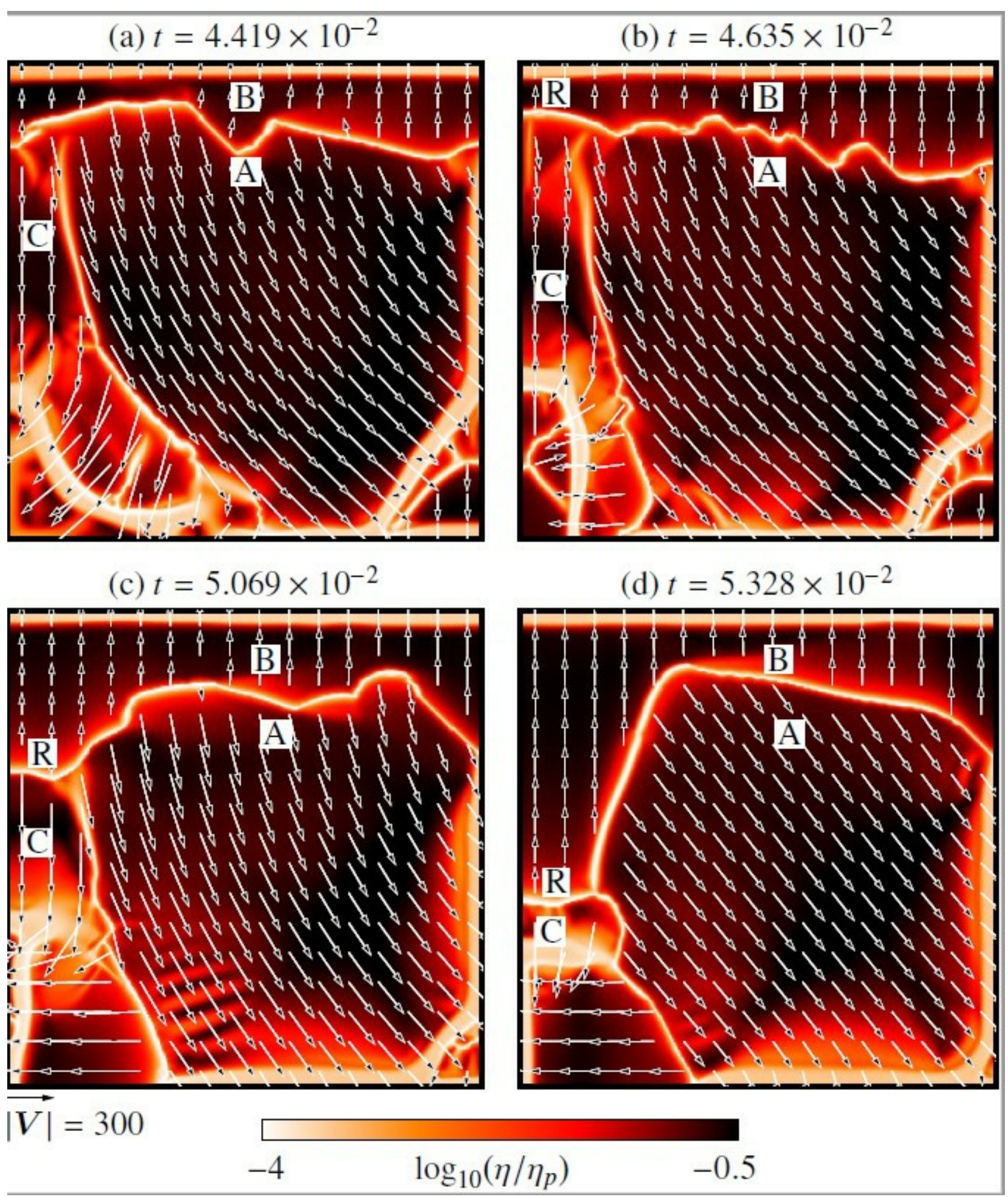

Figure 3

The time evolution of the surface plate motions. Snapshots of the distributions of viscosity $\eta$ (colour) and velocity $V$ (arrows) in a horizontal plane along the surface at the elapsed times indicated in the figure. The values of $\eta$ are normalised by $\eta p$, the viscosity of intact material at the surface. 


\section{Supplementary Files}

This is a list of supplementary files associated with this preprint. Click to download.

manuscript4eps.tex

gia4eps.png

supplement4eps.pdf

spbasic.bst

manuscript4eps.bib

eps.cls 